\title{
SERRE THEOREM FOR INVOLUTORY HOPF ALGEBRAS
}

\author{
G. MILITARU
}

\begin{abstract}
We call a monoidal category $\mathcal{C}$ a Serre category if for any $C, D \in \mathcal{C}$ such that $C \otimes D$ is semisimple, $C$ and $D$ are semisimple objects in $\mathcal{C}$. Let $H$ be an involutory Hopf algebra, $M, N$ two $H$-(co)modules such that $M \otimes N$ is (co)semisimple as a $H$ (co)module. If $N$ (resp. $M$ ) is a finitely generated projective $k$-module with invertible Hattory-Stallings rank in $k$ then $M$ (resp. $N$ ) is (co)semisimple as a $H$-(co)module. In particular, the full subcategory of all finite dimensional modules, comodules or YetterDrinfel'd modules over $H$ the dimension of which is invertible in $k$ are Serre categories.
\end{abstract}

\section{INTRODUCTION}

Around 1950 C. Chevalley [2, pg. 18] proved a remarkable result: if $k$ is a field of characteristic zero, $G$ an abitrary group and $M, N$ two finite dimensional semisimple $k[G]$-modules then $M \otimes N$ is a semisimple $k[G]$-module. Although the result is a purely algebraic one, the only proof known up to date is the one given by Chevalley in which special techniques from algebraic geometry and Lie algebras are used (cf. [6, pg. 16]). The generalization of the Chevalley theorem in positive characteristic was given by J.P. Serre in [4] and the converse in [5, Theorem 2.4]. In fact, we can formulate these properties in the more general setting of monoidal categories:

Local Chevalley-Serre Property: Let $(\mathcal{C}, \otimes, I)$ be a monoidal category and $C, D$ two objects of $\mathcal{C}$. Give a necessary and sufficient condition such that $C \otimes D$ is a semisimple object of $\mathcal{C}$.

Starting from this problem we can derive the following concepts: a monoidal category $\mathcal{C}$ is called a Serre category if for any $C, D \in \mathcal{C}$ such that $C \otimes D$ is semisimple, $C$ and $D$ are semisimple objects in $\mathcal{C}$. A monoidal category $(\mathcal{C}, \otimes, I)$ is called a Chevalley category if $C \otimes D$ is a semisimple object for any semisimple objects $C, D$ of $\mathcal{C}$. The category of all finite dimensional representations of a group $G$ over a field of characteristic zero is the basic example of a Chevalley and Serre category.

This note is dedicated to Serre categories. Proposition 1.1 gives a general example of a Serre categories: a monoidal category $\mathcal{C}$ such that any object has a strong dual and the functors $C \otimes-: \mathcal{C} \rightarrow \mathcal{C},-\otimes C: \mathcal{C} \rightarrow \mathcal{C}$ preserve the monomorphisms for all $C \in \mathcal{C}$ is a Serre category. Together with a couple of technical lemmas presented further on, this result leads to the main Theorem 1.4. In particular, if $k$ is a field and $H$ an involutory

2000 Mathematics Subject Classification. 16W30, 16S40.

Key words and phrases. Hopf algebras, semisimple modules, Chevalley property.

This work was supported by CNCSIS grant 24/28.09.07 of PN II "Groups, quantum groups, corings and representation theory". 
Hopf algebra, then the full subcategory of all finite dimensional modules, comodules or Yetter-Drinfel'd modules over $H$ the dimension of which is invertible in $k$ are Serre categories.

We recall that a morphism $f: C \rightarrow D$ in a category $\mathcal{C}$ is called a split mono if there exists $g: D \rightarrow C$ a morphism in $\mathcal{C}$ such that $g \circ f=\operatorname{Id}_{C}$. The composition of two split monos is a split mono and if $h \circ f$ is a split mono then $f$ is a split mono. An object $D \in \mathcal{C}$ is called semisimple if any monomorphism $f: C \rightarrow D$ is a split mono.

Throughout this note $k$ will be a commutative ring and all modules, Hopf algebras, tensor products and homomorphisms are over $k$. A $k$-module $N$ is finitely generated projective if and only if there exists a (unique) element $R_{N}=\sum_{i=1}^{n} e_{i} \otimes e_{i}^{*} \in N \otimes N^{*}$, called the canonical element of $N$ (or, by abuse of language, dual basis of $N$ ) such that

$$
\sum_{i=1}^{n} e_{i}^{*}(n) e_{i}=n \quad \text { and } \quad \sum_{i=1}^{n} n^{*}\left(e_{i}\right) e_{i}^{*}=n^{*}
$$

for all $n \in N$ and $n^{*} \in N^{*}$. We denote by $r_{k}(N)=\sum_{i=1}^{n} e_{i}^{*}\left(e_{i}\right) \in k$ the (HattoryStallings) rank of $N$; if $k$ is a field then $r_{k}(N)$ is exactly $\operatorname{dim}(N) 1_{k}$. For a Hopf algebra $H$ we will extensively use Sweedler's sigma-notation: $\Delta(h)=h_{(1)} \otimes h_{(2)} \in H \otimes H$ (summation understood). $H$ is called involutory if $S^{2}=\operatorname{Id}_{H} \cdot\left({ }_{H} \mathcal{M}, \otimes, k\right)$ will be the monoidal category of left $H$-modules and $H$-linear maps: $k$ is a left $H$-module via the trivial action $h \cdot a=\varepsilon(h) a$, for all $h \in H$ and $a \in k$ and if $M$ and $N$ are two left $H$-modules $M \otimes N$ is a left $H$-module via the diagonal map: $h \cdot(m \otimes n):=h_{(1)} m \otimes h_{(2)} n$, for all $h \in H, m \in M$ and $n \in N$. The $k$-linear dual $N^{*}=\operatorname{Hom}(N, k)$ is a left $H$-module via

$$
<h \cdot n^{*}, n>:=<n^{*}, S(h) n>
$$

for all $h \in H, n^{*} \in N^{*}$ and $n \in N .\left(\mathcal{M}^{H}, \otimes, k\right)$ will be the monoidal category of right $H$-comodules and $H$-colinear maps. For a right $H$-comodule $(M, \rho) \in \mathcal{M}^{H}$ we denote the coaction by $\rho(m)=m_{<0>} \otimes m_{<1>}$ (summation understood), for all $m \in M$. If $M$ and $N$ are right $H$-comodule then $M \otimes N$ is a right $H$-comodule via $\rho(m \otimes n)=$ $m_{<0>} \otimes n_{<0>} \otimes m_{<1>} n_{<1>}$, for all $m \in M, n \in N$. We recall that a right $H$-comodule is called cosemisimple if it is a semisimple object in the category $\mathcal{M}^{H}$. By $H$-module (resp. $H$-comodule) we mean a left $H$-module (resp. a right $H$-comodule). ${ }_{H} \mathcal{Y} D^{H}$ will be the monoidal category of (left-right) Yetter-Drinfel'd modules: an object of it is at once a left $H$-module and a right $H$-comodule such that

$$
h_{(1)} m_{<0>} \otimes h_{(2)} m_{<1>}=\left(h_{(2)} m\right)_{<0>} \otimes\left(h_{(2)} m\right)_{<1>} h_{(1)}
$$

for all $h \in H$ and $m \in M$. The morphisms of ${ }_{H} \mathcal{Y} D^{H}$ are $H$-linear and $H$-colinear maps.

\section{Serre Theorem for involutory Hopf algebras}

Let $(\mathcal{C}, \otimes, I)$ be a monoidal category. We shall say that an object $D \in \mathcal{C}$ has a strong right dual if there exists an object $D^{*} \in \mathcal{C}$ and a split mono $i_{D}: I \rightarrow D \otimes D^{*}$ in $\mathcal{C}$. An object $C \in \mathcal{C}$ has a strong left dual if there exists an object $C^{*} \in \mathcal{C}$ and a split mono $i_{C}: I \rightarrow C^{*} \otimes C$ in $\mathcal{C}$. A monoidal category $(\mathcal{C}, \otimes, I)$ is called a Serre category if for any 
$C, D \in \mathcal{C}$ such that $C \otimes D$ is semisimple we have that $C$ and $D$ are semisimple objects in $\mathcal{C}$.

Proposition 1.1. Let $(\mathcal{C}, \otimes, I)$ be a monoidal category, $C$ and $D$ two objects in $\mathcal{C}$ such that $C \otimes D$ is a semisimple object in $\mathcal{C}$. The following holds:

(1) If $D$ has a strong right dual and the functor $-\otimes D: \mathcal{C} \rightarrow \mathcal{C}$ preserves monomorphisms then $C$ is semisimple in $\mathcal{C}$.

(2) If $C$ has a strong left dual and the functor $C \otimes-: \mathcal{C} \rightarrow \mathcal{C}$ preserves monomorphisms then $D$ is semisimple in $\mathcal{C}$.

In particular, if any object of $\mathcal{C}$ has strong left and right duals and the functors $C \otimes-$ : $\mathcal{C} \rightarrow \mathcal{C},-\otimes C: \mathcal{C} \rightarrow \mathcal{C}$ preserve monomorphisms for all $C \in \mathcal{C}$ then $\mathcal{C}$ is a Serre category.

Proof. 1) Let $j: C^{\prime} \rightarrow C$ be a monomorphism in $\mathcal{C}$. Then $j \otimes \operatorname{Id}_{D}: C^{\prime} \otimes D \rightarrow C \otimes D$ is a monomorphism in $\mathcal{C}$, hence is a split mono as $C \otimes D$ is semisimple. Consider the commutative diagram

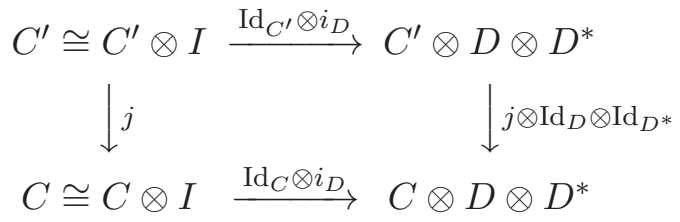

Now, $\operatorname{Id}_{C^{\prime}} \otimes i_{D}$ and $j \otimes \operatorname{Id}_{D} \otimes \operatorname{Id}_{D^{*}}$ are split monos; hence $\left(\operatorname{Id}_{C} \otimes i_{D}\right) \circ j$ is a split mono, thus $j$ is a split mono i.e. $C$ is semisimple.

2) Let $j: D^{\prime} \rightarrow D$ be a monomorphism in $\mathcal{C}$. Then $\operatorname{Id}_{C} \otimes j: C \otimes D^{\prime} \rightarrow C \otimes D$ is a monomorphism in $\mathcal{C}$, hence it is a split mono as $C \otimes D$ is semisimple. Consider the commutative diagram

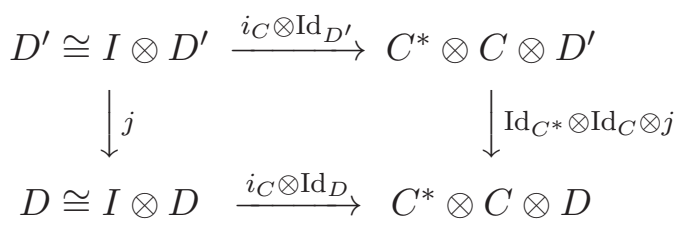

where $i_{C} \otimes \operatorname{Id}_{D^{\prime}}$ and $\operatorname{Id}_{C^{*}} \otimes \operatorname{Id}_{C} \otimes j$ are split monos; hence $\left(i_{C} \otimes \operatorname{Id}_{D}\right) \circ j$ is a split mono, thus $j$ is a split mono i.e. $D$ is semisimple.

In order to give the main examples of Serre categories we need some technical results:

Lemma 1.2. Let $H$ be a Hopf algebra, $N$ be a right $H$-comodule, finitely generated and projective over $k$. Then $N^{*}=\operatorname{Hom}(N, k)$ has a structure of right $H$-comodule via the coaction given by

$$
\rho_{N^{*}}\left(e_{i}^{*}\right)=\sum_{j=1}^{n} e_{j}^{*} \otimes S\left(\left(e_{j}\right)_{<1>}\right) e_{i}^{*}\left(\left(e_{j}\right)_{<0>}\right)
$$

for all $i=1, \cdots, n$, where $\sum_{i=1}^{n} e_{i} \otimes e_{i}^{*} \in N \otimes N^{*}$ is the canonical element of $N$. 
Proof. This is a well-know structure and the proof is just a straightforward verification. Using the $\sum$-notation the coaction given by (3) can be written as follows:

$$
\rho_{N^{*}}(f)=f_{<0>} \otimes f_{<1>} \in N^{*} \otimes H \quad \text { iff } \quad f_{<1>} f_{<0>}(n)=S\left(n_{<1>}\right) f\left(n_{<0>}\right)
$$

for all $n \in N$ and $f \in N^{*}$.

Let $N \in{ }_{H} \mathcal{Y} D^{H}$ be a Yetter-Drinfel'd module, finitely generated and projective over $k$; then $N^{*}=\operatorname{Hom}(N, k) \in{ }_{H} \mathcal{Y} D^{H}$ via the $H$-module structure given by (2) and the $H$-comodule structure given by (3): this is the left-right version of [3, Proposition 4.4.2].

Proposition 1.3. Let $H$ be an involutory Hopf algebra. Then:

(1) Any H-module (resp. $H$-comodule) that is finitely generated projective as a $k$ module with invertible rank in $k$ has a right and a left strong dual in the category ${ }_{H} \mathcal{M}\left(\right.$ resp. $\left.\mathcal{M}^{H}\right)$.

(2) Any Yetter-Drinfel'd module that is finitely generated projective as a $k$-module with invertible rank in $k$ has a right and a left strong dual in the category ${ }_{H} \mathcal{Y} D^{H}$.

Proof. 1) Let $N$ be a $H$-module that is finitely generated projective as a $k$-module with invertible rank $r_{k}(N)$ in $k$. We shall prove that $N^{*}$ with the structure given by (2) is a strong right and left dual of $N$ in ${ }_{H} \mathcal{M}$. We view the canonical element $R_{N}$ as a $k$-linear map

$$
i_{N}: k \rightarrow N \otimes N^{*}, \quad i_{N}(a):=a R_{N}=a \sum_{i=1}^{n} e_{i} \otimes e_{i}^{*}
$$

for all $a \in k$ and we shall prove that $i_{N}$ is an $H$-module map, i.e. we have to prove that

$$
\varepsilon(h) \sum_{i=1}^{n} e_{i} \otimes e_{i}^{*}=\sum_{i=1}^{n} h_{(1)} e_{i} \otimes h_{(2)} \cdot e_{i}^{*}
$$

for all $h \in H$. We shall use the $k$-linear isomorphism

$$
\varphi_{N}: N \otimes N^{*} \rightarrow \operatorname{End}(N), \quad \varphi_{N}\left(n \otimes n^{*}\right)\left(n^{\prime}\right):=<n^{*}, n^{\prime}>n
$$

given by the fact that $N$ is finitely generated projective over $k$. For $n \in N$ we have:

$$
\varphi_{N}\left(\varepsilon(h) \sum_{i=1}^{n} e_{i} \otimes e_{i}^{*}\right)=\varepsilon(h) \sum_{i=1}^{n} e_{i}^{*}(n) e_{i} \stackrel{\underline{\underline{1}}}{=} \varepsilon(h) n
$$

and

$$
\begin{aligned}
\varphi_{N}\left(\sum_{i=1}^{n} h_{(1)} e_{i} \otimes h_{(2)} \cdot e_{i}^{*}\right)(n) & =\sum_{i=1}^{n}\left(h_{(2)} \cdot e_{i}^{*}\right)(n) h_{(1)} e_{i} \\
& \stackrel{20}{=} \sum_{i=1}^{n} h_{(1)} e_{i}^{*}\left(S\left(h_{(2)}\right) n\right) e_{i} \\
& \stackrel{11}{=} h_{(1)} S\left(h_{(2)}\right) n=\varepsilon(h) n
\end{aligned}
$$


hence (44) holds and $i_{N}: k \rightarrow N \otimes N^{*}$ is $H$-linear. Let us consider now the evaluation map

$$
e v_{N}: N \otimes N^{*} \rightarrow k, \quad e v_{N}\left(n \otimes n^{*}\right)=<n^{*}, n>
$$

for all $n \in N$ and $n^{*} \in N^{*}$. As $H$ is a involutory Hopf algebra, $e v_{N}$ is a $H$-linear map. Indeed,

$$
\begin{array}{rlrl}
e v_{N}\left(h \cdot\left(n \otimes n^{*}\right)\right) & = & e v_{N}\left(h_{(1)} n \otimes h_{(2)} \cdot n^{*}\right) \\
& = & & <h_{(2)} \cdot n^{*}, h_{(1)} n> \\
& = & & <n^{*}, S\left(h_{(2)}\right) h_{(1)} n> \\
(S & \stackrel{S^{-1}}{=} & & <n^{*}, \varepsilon(h) n> \\
& = & & \varepsilon(h) e v_{N}\left(n \otimes n^{*}\right) \\
& = & h \cdot e v_{N}\left(n \otimes n^{*}\right)
\end{array}
$$

for all $h \in H, n \in N$ and $n^{*} \in N^{*}$. Thus we have two $H$-module maps

$$
i_{N}: k \rightarrow N \otimes N^{*}, \quad e v_{N}: N \otimes N^{*} \rightarrow k
$$

such that

$$
e v_{N} \circ i_{N}=r_{k}(N)
$$

As $r_{k}(N)$ is invertible in $k$ the map $i_{N}: k \rightarrow N \otimes N^{*}$ is a split mono in the category ${ }_{H} \mathcal{M}$ : the $H$-linear retraction follows from (5) and is given by $r_{k}(N)^{-1} e v_{N}: N \otimes N^{*} \rightarrow k$. Thus $N^{*}$ is a strong right dual of $N$ in ${ }_{H} \mathcal{M}$.

We shall prove now that $N^{*}$ is also a strong left dual of $N$ in ${ }_{H} \mathcal{M}$. We consider the $k$-linear map $j_{N}: k \rightarrow N^{*} \otimes N, j_{N}(a):=a \sum_{i=1}^{n} e_{i}^{*} \otimes e_{i}$, for all $a \in k$. Using the fact that $H$ is an involutory Hopf algebra we can easily show that $i_{N}$ is an $H$-module map: here we use the $k$-linear isomorphism

$$
\psi_{N}: N^{*} \otimes N \rightarrow \operatorname{End}(N), \quad \psi_{N}\left(n^{*} \otimes n\right)\left(n^{\prime}\right):=<n^{*}, n^{\prime}>n
$$

given by the fact that $N$ is finitely generated and projective over $k$. As $r_{k}(N)$ is invertible in $k$ the map $j_{N}: k \rightarrow N^{*} \otimes N$ is split mono in ${ }_{H} \mathcal{M}$ : the $H$-linear retraction is given by $r_{k}(M)^{-1} e v_{N}^{\prime}: N^{*} \otimes N \rightarrow k$, where $e v_{N}^{\prime}: N^{*} \otimes N \rightarrow k, \quad e v_{N}^{\prime}\left(n^{*} \otimes n\right):=<n^{*}, n>$, for all $n^{*} \in N^{*}, n \in N$ is the evaluation map and it is an $H$-module map.

We shall consider now the comodule case. Let $N$ be an $H$-comodule that is finitely generated projective as a $k$-module with invertible rank $r_{k}(N)$ in $k$. We shall prove that $N^{*}$ with the structure given by (3) is a strong right and left dual of $N$ in $\mathcal{M}^{H}$. First we prove that $i_{N}: k \rightarrow N \otimes N^{*}, i_{N}(a)=a \sum_{i=1}^{n} e_{i} \otimes e_{i}^{*}$, for all $a \in k$ is an $H$-comodule map, where $k$ is an $H$-comodule via the trivial coaction $\rho(a)=a \otimes 1_{H}$. Thus we have to prove the formula:

$$
\sum_{i=1}^{n}\left(e_{i}\right)_{<0>} \otimes\left(e_{i}^{*}\right)_{<0>} \otimes\left(e_{i}\right)_{<1>}\left(e_{i}^{*}\right)_{<1>}=\sum_{i=1}^{n} e_{i} \otimes e_{i}^{*} \otimes 1_{H}
$$

If we apply $\rho$ in (1) we obtain

$$
x_{<0>} \otimes x_{<1>}=\sum_{i=1}^{n} e_{i}^{*}(x)\left(e_{i}\right)_{<0>} \otimes\left(e_{i}\right)_{<1>}
$$


for all $x \in N$. We denote LHS the left hand side of (6). We have:

$$
\begin{aligned}
\text { LHS } & \stackrel{\text { (3) }}{=} \sum_{i, j=1}^{n}\left(e_{i}\right)_{<0>} \otimes e_{j}^{*} \otimes\left(e_{i}\right)_{<1>} S\left(\left(e_{j}\right)_{<1>}\right) e_{i}^{*}\left(\left(e_{j}\right)_{<0>}\right) \\
& =\sum_{i, j=1}^{n} e_{i}^{*}\left(\left(e_{j}\right)_{<0>}\right)\left(e_{i}\right)_{<0>} \otimes e_{j}^{*} \otimes\left(e_{i}\right)_{<1>} S\left(\left(e_{j}\right)_{<1>}\right) \\
& \stackrel{77}{\equiv} \sum_{j=1}^{n}\left(e_{j}\right)_{<0>} \otimes e_{j}^{*} \otimes\left(e_{j}\right)_{<1>} S\left(\left(e_{j}\right)_{<2>}\right) \\
& =\sum_{j=1}^{n}\left(e_{j}\right)_{<0>} \otimes e_{j}^{*} \otimes \varepsilon\left(\left(e_{j}\right)_{<1>}\right) 1_{H} \\
& =\sum_{j=1}^{n} e_{j} \otimes e_{j}^{*} \otimes 1_{H}
\end{aligned}
$$

hence (6) holds i.e. $i_{N}: k \rightarrow N \otimes N^{*}$, is an $H$-comodule map. Using the fact that $H$ is involutory we shall prove that the map $e v_{N}: N \otimes N^{*} \rightarrow k, e v_{N}\left(n \otimes n^{*}\right)=\left\langle n^{*}, n>\right.$, for all $n \in N, n^{*} \in N^{*}$ is also an $H$-comodule map. This is equivalent to showing that:

$$
<\left(e_{j}^{*}\right)_{<0>},\left(e_{i}\right)_{<0>}>\otimes\left(e_{i}\right)_{<1>}\left(e_{j}^{*}\right)_{<1>}=\delta_{i j} \otimes 1_{H}
$$

for all $i, j=1, \cdots, n$, where $\delta_{i j}$ is the Kronecker sign. We denote LHS the left hand side of (8). We have:

$$
\begin{aligned}
& \text { LHS } \stackrel{3}{=} \sum_{t=1}^{n}<e_{t}^{*},\left(e_{i}\right)_{<0>}>\otimes\left(e_{i}\right)_{<1>} S\left(\left(e_{t}\right)_{<1>}\right) e_{j}^{*}\left(\left(e_{t}\right)_{<0>}\right) \\
& =\quad \sum_{t=1}^{n} 1 \otimes\left(e_{i}\right)_{<1>} S\left(\left(e_{t}\right)_{<1>}\right) e_{j}^{*}\left(<e_{t}^{*},\left(e_{i}\right)_{<0>}>\left(e_{t}\right)_{<0>}\right) \\
& \stackrel{77}{=} \quad 1 \otimes\left(e_{i}\right)_{<2>} S\left(\left(e_{i}\right)_{<1>}\right) e_{j}^{*}\left(\left(e_{i}\right)_{<0>}\right) \\
& \stackrel{\left(S=S^{-1}\right)}{=} 1 \otimes \varepsilon\left(\left(e_{i}\right)_{<1>}\right) 1_{H} e_{j}^{*}\left(\left(e_{i}\right)_{<0>}\right) \\
& =1 \otimes e_{j}^{*}\left(e_{i}\right) 1_{H}=\delta_{i j} \otimes 1_{H}
\end{aligned}
$$

hence (8) holds and the evaluation map $e v_{N}: N \otimes N^{*} \rightarrow k$ is right $H$-colinear. Thus we have two $H$-comodule maps $i_{N}: k \rightarrow N \otimes N^{*}$ and $e v_{N}: N \otimes N^{*} \rightarrow k$ such that $e v_{N} \circ i_{N}=r_{k}(N)$. As $r_{k}(N)$ is invertible in $k$ the map $i_{N}: k \rightarrow N \otimes N^{*}$ is a split mono in the category $\mathcal{M}^{H}$, i.e. $N^{*}$ is a strong right dual of $N$ in $\mathcal{M}^{H}$.

Finally, we have to prove that $N^{*}$ is also a strong left dual of $N$ in $\mathcal{M}^{H}$. The proof is similar to the computations above. As $H$ is involutory and $r_{k}(N)$ is invertible in $k$ the map $j_{N}: k \rightarrow N^{*} \otimes N$ is split mono in $\mathcal{M}^{H}$ : the $H$-colinear retraction is given by $r_{k}(N)^{-1} e v_{N}^{\prime}: N^{*} \otimes N \rightarrow k$, where $e v_{N}^{\prime}: N^{*} \otimes N \rightarrow k, \quad e v_{N}^{\prime}\left(n^{*} \otimes n\right):=<n^{*}, n>$, for all $n^{*} \in N^{*}, n \in N$ is the evaluation map and it is an $H$-comodule map. 
2) If $N \in{ }_{H} \mathcal{Y} D^{H}$ then $N^{*} \in{ }_{H} \mathcal{Y} D^{H}$ is a right and left dual of $N$ in the category of Yetter-Drinfel'd modules: this follows from 1) as the maps $i_{N}: k \rightarrow N \otimes N^{*}$ and $j_{N}: k \rightarrow N^{*} \otimes N$ are split mono as $H$-linear and $H$-colinear maps, i.e. they are split mono in the category ${ }_{H} \mathcal{Y} D^{H}$.

Using Proposition 1.1 and Proposition 1.3 we obtain the main results of this note:

Theorem 1.4. Let $H$ be an involutory Hopf algebra, $M$ and $N$ two $H$-(co)modules such that $M \otimes N$ is (co)semisimple as a $H$-(co)module. The following hold:

(1) If $N$ is finitely generated projective as a $k$-module with invertible rank in $k$ then $M$ is (co)semisimple as a $H$-(co)module.

(2) If $M$ is finitely generated projective as a $k$-module with invertible rank in $k$ then $N$ is (co)semisimple as a $H$-(co)module.

In particular, if $k$ is a field, the full subcategory of all finite dimensional $H$-modules (reps. H-comodules) that have invertible dimension in $k$ is a Serre category.

Proof. Proposition 1.3 shows that the hypotheses of Proposition 1.1 are true: we note that for a projective $k$-module $X$ the functors $X \otimes-,-\otimes X$ preserve monomorphisms.

Similarly we have obained the following:

Theorem 1.5. Let $H$ be an involutory Hopf algebra, $M$ and $N$ two Yetter-Drinfel'd modules such that $M \otimes N$ is a semisimple object of ${ }_{H} \mathcal{Y} D^{H}$. The following hold:

(1) If $N$ is finitely generated projective as a $k$-module with invertible rank in $k$ then $M$ is a semisimple object of ${ }_{H} \mathcal{Y} D^{H}$.

(2) If $M$ is finitely generated projective as a $k$-module with invertible rank in $k$ then $N$ is a semisimple object of ${ }_{H} \mathcal{Y} D^{H}$.

In particular, if $k$ is a field, the full subcategory of all finite dimensional Yetter-Drinfel'd modules that have invertible dimension in $k$ is a Serre category.

More difficult is the converse of the last statement of Theorem 1.4, which is a generalization of the Chevalley theorem for involutory Hopf algebras (we only state this for characteristic zero):

Question: Let $k$ be a field of characteristic zero, $H$ an involutory Hopf algebra and $M$ and $N$ two finite dimensional (co)semisimple $H$-(co)modules. Is $M \otimes N$ (co)semisimple as a $H$-(co)module?

The answer is affirmative for $H=k[G]$ based on the Chevalley theorem and the fact that $k[G]$ is cosemisimple as a coalgebra. In the case that $H$ is finite dimensional: an involutory finite dimensional Hopf algebra $H$ is semisimple and cosemisimple following the well known theorem of Larson and Radford. In generally, the Chevalley theorem fails for arbitrary finite-dimensional Hopf algebras, an example being the Frobenius-Lusztig kernels $([1])$. 


\section{REFERENCES}

[1] N. Andruskiewitsch, P. Etingof, and S. Gelaki, Triangular Hopf algebras with the Chevalley property, Michigan Math. J. 49 (2001), 277-298.

[2] C. Chevalley, Theorie des Groupes de Lie, Vol. III, Hermann, Paris, 1954.

[3] L. A. Lambe and D. Radford, Algebraic aspects of the quantum Yang-Baxter equation, J. Algebra 154 (1992) 222-288

[4] J.-P. Serre, Sur la semi-simplicite des produits tensoriels de representations de groupes, Invent. Math. 116(1994), 513-530.

[5] J.-P. Serre, Semisimplicity and Tensor Products of Group Representations: Converse Theorems, J. Algebra 194 (1997) 496-520.

[6] J.-P. Serre, Moursund Lectures 1998, Notes by W.E. Duckworth, availabe at http://math.uoregon.edu/resources/serre/

Faculty of Mathematics and Computer Science, University of Bucharest, Str. Academiei 14, RO-010014 Bucharest 1, Romania

E-mail address: gigel.militaru@fmi.unibuc.ro and gigel.militaru@gmail.com 